American J. of Engineering and Applied Sciences 4 (4): 556-565, 2011

ISSN 1941-7020

(C) 2014 S. Aliesawi et al., This open access article is distributed under a Creative Commons Attribution

(CC-BY) 3.0 license

\title{
Adaptive Channel Estimation for Multiuser Underwater Communication Systems
}

\author{
Aliesawi, S., C.C. Tsimenidis, B.S. Sharif and M. Johnston \\ School of Electrical, Electronics and Computer Engineering, \\ Newcastle University, Newcastle upon Tyne, NE1 7RU, UK
}

\begin{abstract}
Problem statement: Multiple Access Interference (MAI) signals and poor estimation of the unknown channel parameters in the presence of limited training sequences are two of the major problems that degrade the systems' performance. Two synchronous multiuser receivers with Rake reception of Interleave Division Multiple Access (IDMA) and Code Division Multiple Access (CDMA) systems, in conjunction with channel estimation, are considered for communication over different short range shallow water acoustic channels. Approach: The proposed hard/soft chip channel estimation and carrier phase tracking are jointly optimized based on the Mean Square Error (MSE) criterion and adapted iteratively by the reconstructed MAI signal. This is generated from exchanged soft information in terms of Log-Likelihood Ratio (LLR) estimates from the single-users' channel decoders. The channel parameters and error estimation are used to enable the chip cancellation process to retrieve an accurate measurement of the detrimental effects of Intersymbol Interference (ISI) and MAI. Results: The performance of the proposed receiver structures with small processing gain are investigated and compare with 2 and 4 synchronous users using memoryless Quadrature Phase-Shift Keying (QPSK) at an effective rate of 439.5 bps per user. Conclusion: The results demonstrate that the performance is limited by MAI and ISI signals and the IDMA performance outperforms long code CDMA and short code CDMA.
\end{abstract}

Key words: Multiuser detection, channel estimation, underwater acoustic communication, rake detectors, Code Division Multiple Access (CDMA), Log-Likelihood Ratio (LLR), Quadrature Phase-Shift Keying (QPSK), Multiple Access Interference (MAI)

\section{INTRODUCTION}

Underwater acoustic communication networks are a technology for the development of ocean communication systems. Since underwater communication uses acoustic waves instead of electromagnetic waves, it is only capable of low data rates compared with terrestrial communication. In such propagation mediums a low speed of sound propagation, time varying multipath channels and limited bandwidth are the main characteristics that result in a poor communication link and high latency posing challenges very different from terrestrial wireless networking. Therefore, due to these properties and bandwidth limitations, accomplishing the same data rate for underwater communication is impractical (Nowsheen et al., 2010). Spread spectrum systems hold significant promise for such acoustic communications. These systems can perform well in multipath environments and offer communication at low Signalto-Noise Ratios (SNRs), but at the expense of a decreased data rate (Stojanovic and Freitag, 2006; Li and Preisig, 2007). In Code Division Multiple Access (CDMA) and Interleave Division Multiple Access (IDMA) networks, Multiuser Detectors (MUD) is used to overcome the channel impairments and Multiple Access Interference (MAI) between users. In CDMA systems, orthogonal or near orthogonal spreading sequences distinguish different users and cause bandwidth expansion without coding gain. Therefore, in order to maximize the power and bandwidth efficiency, IDMA with user specific interleaves has been proposed to meet these requirements by devoting the entire bandwidth expansion to low rate channel coding. It mitigates the MAI and Intersymbol Interference (ISI) jointly by using a low complexity chip strategy (Ping et al., 2006; Zhou et al., 2007), which is far less complex than optimal (Moher, 1998) and suboptimal detectors (Wang and Poor, 1999) used in CDMA systems. The receivers with symbol rate adaptation may not perform satisfactorily in dynamic underwater channels, where the channel variations over symbol duration are not

Corresponding Author: Aliesawi, S., School of Electrical, Electronics and Computer Engineering, Newcastle University, Newcastle upon Tyne, NE1 7RU, UK 
Am. J. Engg. \& Applied Sci., 4 (4): 556-565, 2011

negligible. Therefore, it is desirable for the receiver algorithmc to be able to adapt its coefficients more frequently than a symbol interval, thus offering the possibility to track channel variations associated with large Doppler spreads (Jianguo et al., 2007).

Previous work on chip multiuser detection, e.g. (Ping et al., 2006; Xiong and $\mathrm{Hu}, 2008$ ), are based on the assumption that the receiver has perfect channel estimation. Unfortunately, for doubly selective channels, as in Underwater Acoustic Channels (UACs), proper channel state information estimation becomes very difficult at low SNRs and imperfect channel estimation can significantly degrade performance. Iterative linear channel estimation schemes for IDMA are discussed and compared in (Schoeneich and Hoeher, 2006), where data and training are superimposed to allow channel estimation and detection to be done at the same time. Additionally, an IDMA system integrated with channel estimation is studied in (Zhou et al., 2007), which uses both pilot and soft decoded data in the same way as (Schoeneich and Hoeher, 2006). A method of multipath CDMA channel estimation has been investigated in (Choi, 1999), by utilising pilot and traffic channels. Long code CDMA with orthogonal signalling has been the subject of study in (Strom and Miller, 2002), where the Maximum Likelihood (ML) channel estimator was employed over time varying channels. Iterative soft channel estimation with turbo equalization (Otnes and Tuchler, 2004) was used in (Otnes and Eggen, 2008) over long-term sea trials, where the extended training symbols are generated between the ordinary symbols. Eggen et al. $(2000 ; 2001)$ the linear equalizer combined with separate channel estimation has been proposed and its development in Ping et al. (2006) has investigated the impact of sparse channel estimation on the equalization of phase coherent communication signals.

The low cost chip-by-chip multiuser detection algorithms of IDMA systems have yet to be fully studied, especially for real channel conditions and the effectiveness of these detectors degrades significantly if the channel estimates are not reliable. In this study, an adaptive chip rate channel estimation algorithm in (Aliesawi et al., 2010a; 2010b) with multiplexed training sequence and joint phase tracking are considered for synchronous IDMA and CDMA systems. Channel estimation is refined using both training sequence and enhanced iterative soft feedback information. The performance of the systems is demonstrated using experimental sea-trial data over different ranges.

\section{MATERIALS AND METHODS}

System models: For a packet-wise downlink IDMA transmission in Fig. 1, the information bits, $b_{k}(n)$, of user $\mathrm{k}$ are encoded by a rate $1 / 2$ convolution Encoder (ENC), producing the coded bit sequence.

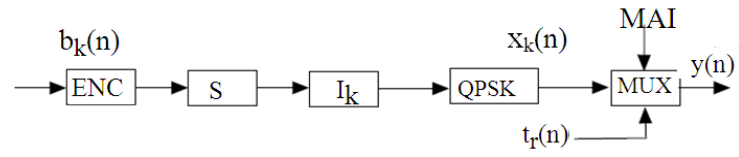

Fig. 1: IDMA downlink transmitter (I and $\mathrm{S}$ denote interleavers and spreaders, respectively)

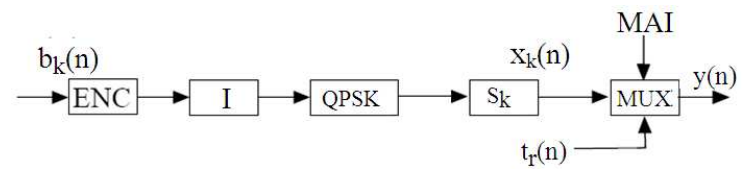

Fig. 2: CDMA downlink transmitter

The sequence is further processed by a length 8 scrambling repetition code (S) and shuffled using pseudo-random user-specific interleavers $I_{k}$. The interleaved bits are then modulated using Quadrature Phase-Shift Keying (QPSK). The coded bits of the CDMA transmitter in Fig. 2, are interleaved by a bitlevel interleaver and modulated using QPSK modulation. The symbols are then up-sampled by 8 and scrambled using a user-specific pseudorandom scrambling sequence $S_{k}$. The training sequence, $t_{r}(n)$, can be any well-known modulation scheme such as Binary Phase Shift Keying (BPSK) or QPSK. In principle, the selection of the modulation scheme is done according to power, bandwidth efficiency and the channel capacity (Tchamkerten and Telatar, 2006; Milewski, 1983). An important property of the multiplexed training sequence is the length of its period. In general, a longer period leads to a better channel estimate. However, the user's signals are summed and multiplexed with a BPSK modulated training sequence, $t_{r}(n)$, to form the transmitted signal $y(t)$. The baseband received signal after transmission over the UAC can be expressed as in Eq. 1:

$$
r(t)=\sum_{l=0}^{L-1} h_{1}(t) y\left(t-\tau_{1}\right) e^{j \phi_{1}(t)}+w(t)
$$

where, $y(t)=\left[\mathrm{t}_{\mathrm{r}}(\mathrm{t}), \sum_{\mathrm{k}=1}^{\mathrm{k}} \mathrm{x}_{\mathrm{k}}(\mathrm{t})\right]$ is the transmitted signal containing all users synchronously combined, $\mathrm{h}_{1}(\mathrm{t})$ are the channel fading coefficients of the physical propagation path 1 at time $t$ and the corresponding path delay is $\tau_{1}$. The total number of paths is $L$ and the phase $\phi_{1}(\mathrm{n})$ represents the joint effects of phase distortion due to motion and carrier offsets. w(t) represents the additive white Gaussian noise samples.

Frame structure and signal acquisition: The signal carrier frequency was $12 \mathrm{kHz}$ band-limited to $4 \mathrm{kHz}$. The transmission was organized in packets of equal duration. Each packet comprised a 511 BPSK equalizer 
training sequence encapsulated with QPSK symbols, 50 ms Linear Frequency Modulated chirp signals (LFM) at the start and end of each frame and $12.5 \mathrm{~ms}$ gap periods. The corresponding effective data rate amounts to 1024 information bits divided by block duration of $2.33 \mathrm{~ms}$ equal to $439.5 \mathrm{bps}$ users. The band pass received signal after passing through the Band Pass Filter (BPF) with carrier frequency $f_{c}$, to remove unwanted low frequencies, is frame synchronized before any processing. The frame synchronization is achieved by matched filtering with a $50 \mathrm{~ms}$ local replica of the chirp signal that has good correlation properties. After the signal is shifted to the complex baseband by employing in-phase and quadrature oscillator mixers, the acquired signal is sampled using one sample per symbol interval and given as an input to the adaptive iterative receiver.

IDMA receiver structure: The soft Rake multiuser detector in Fig. 3a combines the peaks of all paths $(\mathrm{L}+1)$ together with multiple access cancellation concepts to handle the ISI and MAI effects jointly and improve the performance as in Ping et al. (2006). Due to the central limit theorem, the remaining interference $\eta_{\mathrm{k}, 1}(\mathrm{n})$ with respect to user $\mathrm{k}$ is modelled as a Gaussian random variable Eq. 2:

$$
\tilde{\mathrm{r}}(\mathrm{n}+1)=\mathrm{h}_{\mathrm{k}, 1} \mathrm{r}(\mathrm{n}+1)=\left|\mathrm{h}_{\mathrm{k}, 1}^{*}(\mathrm{n})\right|^{2} \mathrm{x}_{\mathrm{k}}(\mathrm{n})+\tilde{\mathrm{\eta}}_{\mathrm{k}, \mathrm{l}}(\mathrm{n})
$$

where, (.)* denotes conjugate operation and interference term is written as Eq. 3:

$$
\tilde{\eta}_{\mathrm{k}, \mathrm{l}}(\mathrm{n})=\mathrm{h}_{\mathrm{k}, \mathrm{l}}(\mathrm{n}) \eta_{\mathrm{k}, \mathrm{l}}(\mathrm{n})
$$

This approximation is sufficiently good especially for a large number of users. However, the Gaussian approximation of the distortion term may be inaccurate as the number of users becomes small. Nevertheless, the benefit of less interference generally offsets the problem due to interference modeling (Ping, 2005). The details of the detector algorithm in multipath complex time varying channels is presented below Ping et al. (2006), where $\mathrm{m}$ (.) and $\mathrm{v}($.$) denote the mean and$ variance respectively. 7):

Interference mean and variance estimation (Eq. 4-

$$
\begin{aligned}
& \mathrm{m}[\mathrm{r}(\mathrm{n})]=\sum_{\mathrm{k}, 1} \mathrm{~h}_{\mathrm{k}, 1}(\mathrm{n}) \mathrm{m}\left[\mathrm{x}_{\mathrm{k}}(\mathrm{n}-1)\right], \forall \mathrm{n} \\
& \operatorname{Cov}[\mathrm{r}(\mathrm{n})]=\sum_{\mathrm{k}, 1} \mathrm{H}_{\mathrm{k}, 1}(\mathrm{n}) \operatorname{Cov}\left[\mathrm{x}_{\mathrm{k}}(\mathrm{n}-1)\right] \\
& \mathrm{H}_{\mathrm{k}, \mathrm{l}}^{\mathrm{T}}(\mathrm{n})+\sigma^{2} \mathrm{~A}, \quad \forall \mathrm{n}
\end{aligned}
$$

$$
\begin{aligned}
& \mathrm{m}\left[\tilde{\eta}_{\mathrm{k}, 1}(\mathrm{n})\right]=\mathrm{h}_{\mathrm{k}, \mathrm{l}}^{*}(\mathrm{n}) \mathrm{m}[\mathrm{r}(\mathrm{n}+\mathrm{l})]- \\
& \left|\mathrm{h}_{\mathrm{k}, 1(\mathrm{n})}\right|^{2} \mathrm{~m}\left[\mathrm{x}_{\mathrm{k}}(\mathrm{n})\right], \forall \mathrm{k}, \mathrm{n} \\
& \mathrm{v}\left[\tilde{\eta}_{\mathrm{k}, 1}(\mathrm{n})\right]=\mathrm{H}_{\mathrm{k}, 1}^{\mathrm{T}}(\mathrm{n})\left[\operatorname { C o v } \left[\mathrm{r}(\mathrm{n}+1)-\mathrm{H}_{\mathrm{k}, 1}(\mathrm{n})\right.\right. \\
& \operatorname{Cov}\left[\mathrm{x}_{\mathrm{k}}(\mathrm{n}+1)\right] \mathrm{H}_{\mathrm{k}, \mathrm{l}}^{\mathrm{T}}(\mathrm{n}), \quad \forall \mathrm{k}, \mathrm{n}
\end{aligned}
$$

LLRs generation and combining (Eq. 8 and 9):

$$
\begin{aligned}
& \mathrm{L}_{\mathrm{M}}\left[\mathrm{x}_{\mathrm{k}}^{\mathrm{I}}(\mathrm{n})\right]_{1}=2\left|\mathrm{~h}_{\mathrm{k}, \mathrm{l}}(\mathrm{n})\right|^{2} \frac{\tilde{\mathrm{r}}^{\mathrm{I}}(\mathrm{n}+1)-\mathrm{m}\left[\tilde{\eta}_{\mathrm{k}, 1}^{\mathrm{I}}(\mathrm{n})\right]}{\mathrm{v}\left[\tilde{\eta}_{\mathrm{k}, \mathrm{l}}^{\mathrm{I}}(\mathrm{n})\right]}, \\
& \forall \mathrm{k}, \mathrm{n}
\end{aligned}
$$

$\mathrm{L}_{\mathrm{M}}\left[\mathrm{x}_{\mathrm{k}}^{\mathrm{I}}(\mathrm{n})\right]=\sum_{\mathrm{l}=0}^{\mathrm{L}-1} \mathrm{~L}_{\mathrm{M}}\left[\mathrm{x}_{\mathrm{k}}^{\mathrm{I}}(\mathrm{n})\right]_{\mathrm{l}}, \forall \mathrm{k}, \mathrm{n}$

where, $\mathrm{A}$ is a $2 \times 2$ identity matrix, $(.)^{\mathrm{T}}$ denotes the transpose operation and channel matrix is written as Eq. 10:

$$
\mathrm{H}_{\mathrm{k}, \mathrm{l}}(\mathrm{n})=\left[\begin{array}{cc}
\mathrm{h}_{\mathrm{k}, 1}^{\mathrm{I}}(\mathrm{n}) & -\mathrm{h}_{\mathrm{k}, 1}^{\mathrm{Q}}(\mathrm{n}) \\
\mathrm{h}_{\mathrm{k}, 1}^{\mathrm{Q}}(\mathrm{n}) & \mathrm{h}_{\mathrm{k}, \mathrm{l}}^{\mathrm{I}}(\mathrm{n})
\end{array}\right]
$$

The a priori means and variances of the transmitted signal are also required, which is calculated as in (Eq. 11-12) in Wang and Poor (1999) based on the extrinsic LLRs of the decoders:

$$
\begin{aligned}
& \mathrm{m}\left[\mathrm{x}_{\mathrm{k}}(\mathrm{n})\right]=\tanh \left(\frac{\mathrm{L}_{\mathrm{e}}\left[\mathrm{x}_{\mathrm{k}}^{\mathrm{I}}(\mathrm{n})\right.}{2}\right)+\mathrm{j} \tanh \left(\frac{\mathrm{L}_{\mathrm{e}}\left[\mathrm{x}_{\mathrm{k}}^{\mathrm{Q}}(\mathrm{n})\right.}{2}\right) \\
& \operatorname{Cov}\left[\mathrm{x}_{\mathrm{k}}(\mathrm{n})\right]=\left[\begin{array}{cc}
1-\mathrm{m}\left[\mathrm{x}_{\mathrm{k}}^{\mathrm{I}}(\mathrm{n})\right] & 0 \\
0 & 1-\mathrm{m}\left[\mathrm{x}_{\mathrm{k}}^{\mathrm{Q}}(\mathrm{n})\right]
\end{array}\right]
\end{aligned}
$$

where, the off-diagonal entries of $\operatorname{Cov}\left(\mathrm{x}_{\mathrm{k}}(\mathrm{n})\right)$ are considered zeros, as the real and imaginary parts $\mathrm{x}_{\mathrm{k}}^{\mathrm{I}}$ and $\mathrm{x}_{\mathrm{k}}^{\mathrm{Q}}$ of $\mathrm{x}_{\mathrm{k}}(\mathrm{n})$ are assumed uncorrelated. The same procedure can be adopted to calculate $\mathrm{L}_{\mathrm{M}}\left[\mathrm{x}_{\mathrm{k}}^{\mathrm{Q}}(\mathrm{n})\right]$.

Adaptive soft/hard channel estimation: The situation in UACs, where the received amplitude and phase randomly vary, requires robust channel estimation for precise ISI and MAI reconstruction. The low cost channel estimation provides the time varying channel coefficients $h(n)$ at each time instant $n$ to be used in the detector. The adaptive channel estimation algorithm aims to minimize the error signal e(n) as Eq. 13: 
Am. J. Engg. \& Applied Sci., 4 (4): 556-565, 2011

$$
\begin{aligned}
e(n) & =r(n)-\hat{r}(n) \\
& =r(n)-\hat{h}^{T}(n) \tilde{y}(n) e^{-j \hat{\varphi}(n)}
\end{aligned}
$$

where, $\hat{\phi}(\mathrm{n})$ denotes a phase correction parameter, $\hat{\mathrm{y}}(\mathrm{n})=[\tilde{\mathrm{y}}(\mathrm{n}), \ldots \tilde{\mathrm{y}}(\mathrm{n}+\mathrm{M}-1)]^{\mathrm{T}}$ is the regenerated MAI signal and $\hat{\mathrm{h}}(\mathrm{n})=[\tilde{\mathrm{h}}(\mathrm{n}), \ldots \tilde{\mathrm{h}}(\mathrm{n}, \mathrm{M}-1)]^{\mathrm{T}}$ represents the estimated channel. The problem with the minimization is that not all the $\tilde{y}(\mathrm{n})$ are known to the receiver. Therefore, the soft/hard estimates of $\tilde{\mathrm{x}}_{\mathrm{k}}(\mathrm{n})$ are used to form $\tilde{y}(n)=\sum_{k} \tilde{x}_{k}(n)$ and incorporated into the channel estimator together with the $t_{r}(n)$. When the training symbols have been transmitted, $\tilde{y}(n)$ is replaced with $t_{r}(n)$.

The minimization of error variance can be simplified by employing an iterative procedure using a stochastic gradient algorithm (Eggen et al., 2001). The Normalized Least Mean Square (NLMS) algorithm is used to minimize Eq. 13 and compute $\hat{\mathrm{h}}(\mathrm{n})$ as Eq. 14 (Haykin, 2002):

$$
\hat{\mathrm{h}}(\mathrm{n}+1)=\hat{\mathrm{h}}(\mathrm{n})+\frac{\mu}{\varepsilon+\|\tilde{y}(\mathrm{n})\|^{2}} \mathrm{e}(\mathrm{n}) \tilde{\mathrm{y}}(\mathrm{n}) \mathrm{e}^{-j \hat{\phi}(\mathrm{n})}
$$

where $0<\mu<2$ represents the adaptation constant, $\varepsilon$ is a small positive constant and $\|\tilde{\mathrm{y}}(\mathrm{n})\|^{2}=\left[\sum_{\mathrm{m}=0}^{\mathrm{M}-1}|\tilde{\mathrm{y}}(\mathrm{n}, \mathrm{m})|^{2}\right]$ is the Euclidean norm of the tap-input vector $\tilde{y}(n)$.

In general, the choice of filter length $M$ is proportional to the channel dimension and it becomes more computational as it covers the overall delay channel spread. A large $\mathrm{M}$ will project too much noise on the channel response estimates as well as increasing the estimation error. In contrast, a small $\mathrm{M}$ will project too little signal energy onto the channel estimates. Additionally, it suffices to keep only the taps with significant amplitude (Ping et al., 2006) to provide complexity reduction. Therefore should be chosen such that the most significant part of the delay spread is covered to maintain the stability of the algorithm and reduce the noise error contribution. This choice should be based on the channel characteristics and the required performance. However, with a long filter length, the assumption about tracking becomes unrealistic if the channel variation is significant. Unfortunately, an acceptable choice is not always available especially when the channel has long delay spread and fluctuates rapidly (Li and Preisig, 2007).
In order to refine the channel estimate, the hard or soft information feedback from the decoders is obtained by mapping coded bits $\mathrm{L}_{\mathrm{e}}\left[\mathrm{x}_{\mathrm{k}}(\mathrm{n})\right]$ from the previous iteration on to the signal constellation. The hard/soft estimates $\tilde{\mathrm{x}}_{\mathrm{k}}(\mathrm{n})$ are generated in the case of QPSK as Eq. 15:

$\tilde{x}_{k}(n)=\operatorname{sgn}\left\{L_{e}\left[x_{k}^{I}(n)\right]\right\}+j \operatorname{sgn}\left\{L_{e}\left[x_{k}^{Q}(n)\right]\right\}$

for hard symbol estimation, where sgn $\{$.$\} represents the$ sign function and Eq. 16:

$$
\tilde{\mathrm{x}}_{\mathrm{k}}(\mathrm{n})=\tanh \left\{\mathrm{L}_{\mathrm{e}}\left[\mathrm{x}_{\mathrm{k}}^{\mathrm{I}}(\mathrm{n})\right] / 2\right\}+\mathrm{j} \tanh \left\{\mathrm{L}_{\mathrm{e}}\left[\mathrm{x}_{\mathrm{k}}^{\mathrm{Q}}(\mathrm{n})\right] / 2\right\}
$$

for soft symbol estimation. Additionally, soft feedback is intuitively better than hard feedback as it improves tracking capability by reducing error propagation due to the relatively large detection amplitude errors of hard decisions for dynamic channels (Otnes and Tuchler, 2004; Song et al., 2004). If the channel is varying slowly, it may be satisfactory to estimate a channel, which is constant over a frame of data; otherwise the channel coefficients should be updated every chip interval.

Carrier phase tracking: The phase update is optimized jointly using a first order Phase Looked Loop (PLL) (Stojanovic and Zvonar, 1996) in Eq. 17:

$$
\hat{\phi}(\mathrm{n}+1)=\hat{\phi}(\mathrm{n})+\alpha \in(\mathrm{n})
$$

where, $\alpha$ is a positive adaptation constant and the $\in(\mathrm{n})$ represents the phase error measurement, defined as Eq. 18:

$\in(\mathrm{n})=\operatorname{Im}\{\mathrm{r} *(\mathrm{n}) \hat{\mathrm{r}}(\mathrm{n})\}$

where, $\operatorname{Im}($.$) refers to the imaginary part. An estimate of$ $\sigma_{\mathrm{w}(\mathrm{n})}^{2}$ can be given using the time average of the squared error in Eq. 19:

$\sigma_{\mathrm{w}(\mathrm{n})}^{2}=\mathrm{E}\left[\mathrm{e}(\mathrm{n})^{*} \mathrm{e}(\mathrm{n})\right]$

Joint channel estimation and turbo process: In the first iteration, where the errors usually exist since no decisions fed back are available, a non-iterative MUD and training sequence $\mathrm{t}_{\mathrm{r}}(\mathrm{n})$, can be applied to obtain an initial estimate of the complex channel coefficients. Alternatively, a hypothetical transmit sequence $\tilde{\mathrm{x}}_{\mathrm{k}}(\mathrm{n})$ is considered, where the non-training symbols are made up of a sequence of symbols in $(+1,-1)$ as in ( $\mathrm{Li}$ et al., 2007; Flanagan and Fagan, 2007), since initializing to the zero vector would require a larger number of iterations and higher computation cost. 


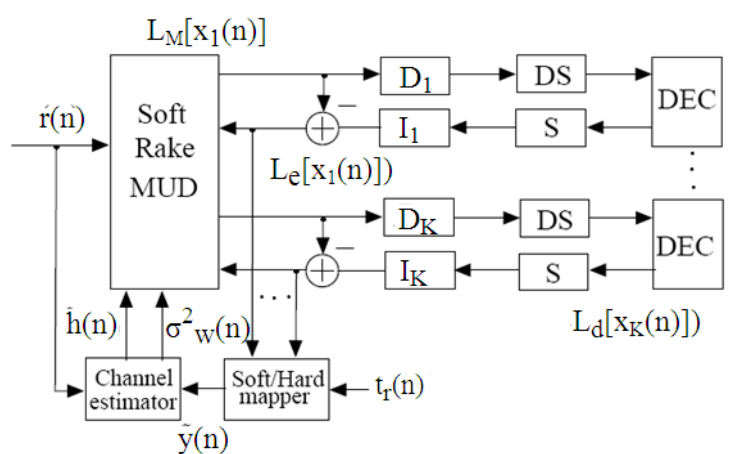

(a)

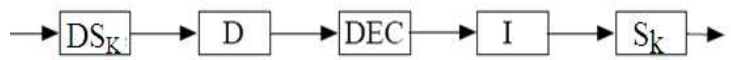

(b)

Fig. 3: IDMA Receiver in (a) can be interpreted as CDMA by exchanging the order of $\mathrm{I}$ and $\mathrm{S}$ as in (b) (D and DS denote deinterleavers and desperadoes, respectively)

This estimate can then be used to start the equalization and detection and compute the LLRs after removing the training sequence.

The detection process is used to reconstruct the channel symbols and mitigate the ISI and MAI jointly using a Parallel Interference Cancellation (PIC) approach. The LLRs after detection are passed through deinterleavers, despreaders blocks and channel decoders. The decoders generate the LLRs of the coded bits $\mathrm{L}_{\mathrm{d}}\left[\mathrm{x}_{\mathrm{k}}(\mathrm{n})\right]$ for exchange after interleaving with iterative detection and channel estimation. These feedback decisions in the form of extrinsic information are assumed to be correct.

Therefore, the hypothetical sequence is replaced by $\tilde{y}_{k}(n)$, after the hard/soft feedback decision of Eq. 15 or 16 , respectively, to refine the channel estimation. Repeating the above procedure can improve the performance with enhanced channel tracking capability and after a number of iterative operations, a hard decision is performed on the decoded data.

Adaptive Chip-Level CDMA Receiver: Most existing multiuser detection CDMA algorithms usually (e.g. in (Widrow et al., 1975; Wang and Poor, 1999)) operate at symbol level, unlike the chip detection strategy in the IDMA system. The CDMA receiver is re-formulated in a similar way to the IDMA receiver in Fig. 3a. Although the chip detection strategy is developed for IDMA systems, it can be directly applied to CDMA systems as well (Nagy et al., 2008; Krzymien et al.,
2008). By doing so, CDMA is only a special case of IDMA and the differences between them are illuminated. CDMA separates multiple users using different scrambling codes, which are random and differ from symbol to symbol.

Additionally, CDMA places the interleavers between the despreaders and decoders as in Fig. 3b, operating at the bit level and are used to alleviate the fading effect. The CDMA receivers rely on a chip detection approach and avoid the matrix operations in (Wang and Poor, 1999), which are needed to handle the correlation among the user specific sequences.

\section{RESULTS}

Experiment setup and channel characteristics: Experimental data was recorded and processed offline. The recorded signals were acquired during sea trials carried out by Newcastle University in the summer of 2009 in the North Sea, a few miles off the coast. Each system is simulated with 2 and 4 simultaneous users over three different channels ranges. All the interleaves are generated independently and randomly from a uniform distribution. The repetition code adopts the same spreading sequence in the IDMA system, i.e. $\{+1,-1,+1 ;-1,+1,-1,+1,-1\}$, for all users. The users' spreading sequences in the investigated CDMA systems are also randomly generated. In short code CDMA packets, the signatures assigned to users during simulation are listed in Table 1 and they are periodic or remain the same for all symbols.

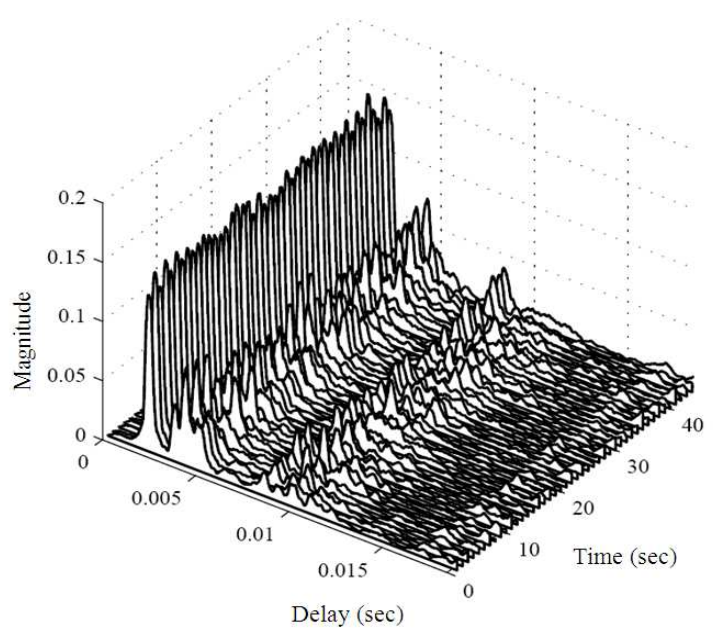

Fig. 4: Normalized channel impulse responses profile of $1000 \mathrm{~m}$ channel range 


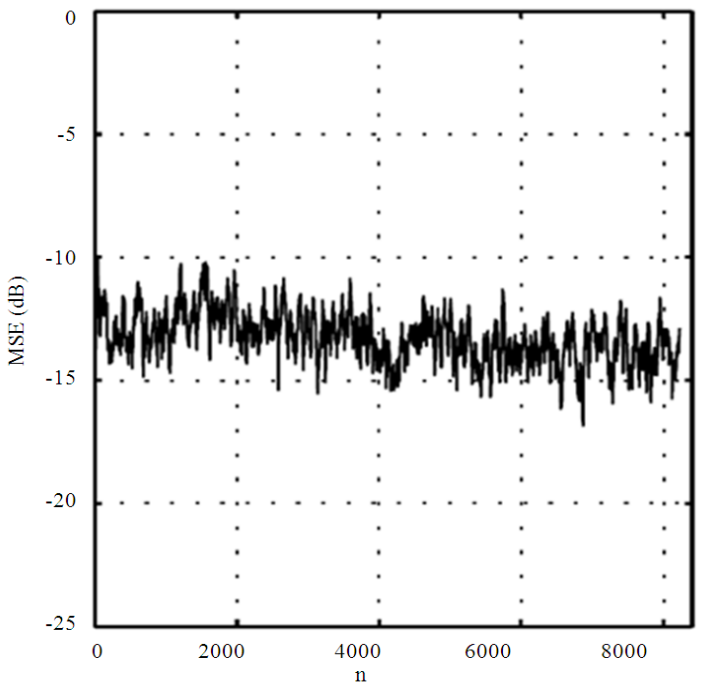

(a)

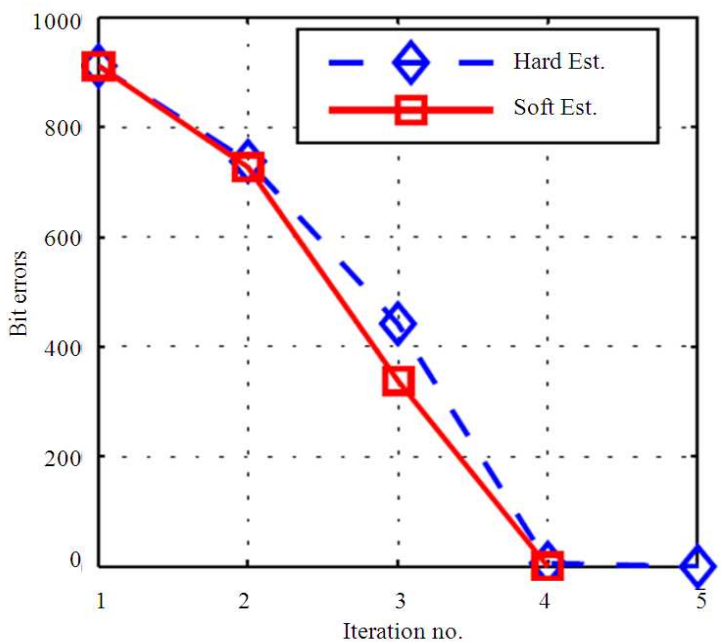

(b)

Fig. 5: IDMA results, Bit errors $=0, \operatorname{SINR}=7.8(\mathrm{~dB})$, $(\mathrm{K}, \mathrm{M}, \mu, \alpha)=(2,16,0.1,0.0001)$ (a) Meansquared error: (b) Bit errors versus iteration

In long code CDMA, they are periodic or essentially pseudorandom and vary for different 1028 transmitted symbols. The setup ranges between the transmitter and the receiver were 200,500 and $1000 \mathrm{~m}$. The channel impulse response at $1000 \mathrm{~m}$ channel range is shown in Fig. 4. In the Table 2, the total numbers of bit errors averaged over 15 packets for IDMA, long-code CDMA and short-code CDMA are given, along with the achieved average output SINR in $\mathrm{dB}$, where the level of convergence represents an approximation of the SINR at the output of the detector (Proakis and Salehi, 2008).

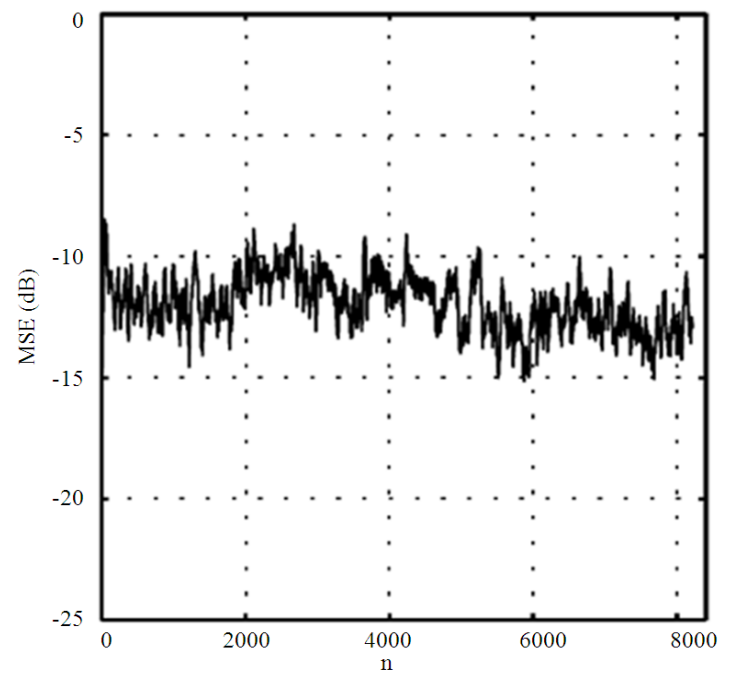

(a)

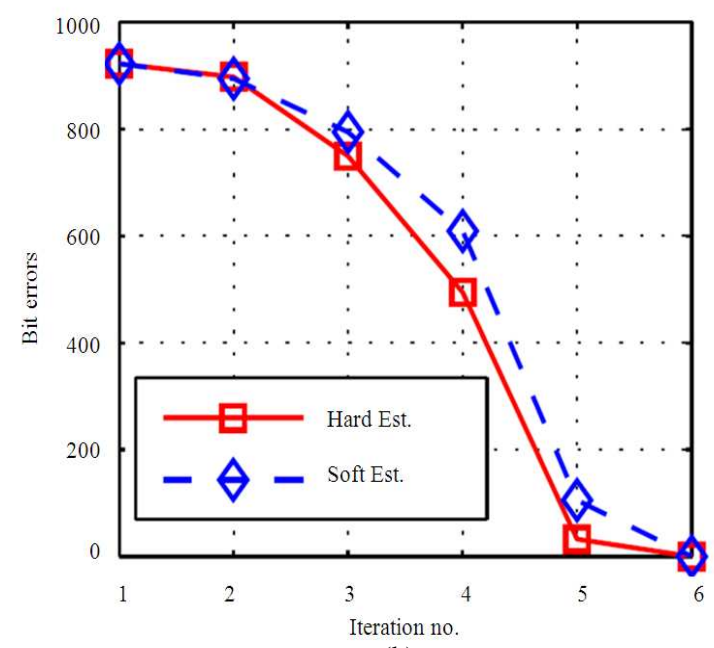

(b)

Fig. 6: Long-code CDMA results: $B$ it errors $=0$, $\mathrm{SINR}=7.2(\mathrm{~dB}),(\mathrm{K}, \mathrm{M}, \mu, \alpha)=(2,16,0.1$, 0.0001) (a) Mean-squared error: (b) Bit errors versus iteration.

Table 1: Spreading sequences of short code CDMA

\begin{tabular}{lc}
\hline User number & Signature sequence \\
\hline 1 & $+1,-1,+1,+1,+1,+1,+1,+1$ \\
2 & $-1,-1,+1,-1,-1,-1,-1,-1$ \\
3 & $+1,+1,+1,-1,-1,-1,-1,+1$ \\
4 & $-1,-1,+1,-1,-1,-1,-1,-1$ \\
\hline
\end{tabular}

For a two-user scenario $(K=2)$, the MSE of the algorithm at the last iteration is drawn in Fig. 5a-7a, along with the receiver parameters of packet no. 1 over $200 \mathrm{~m}$ channel range. 
Am. J. Engg. \& Applied Sci., 4 (4): 556-565, 2011

Table 2: Performance results

\begin{tabular}{|c|c|c|c|c|c|c|c|c|c|}
\hline \multirow[b]{2}{*}{ Channel Range(m) } & \multicolumn{3}{|l|}{ IDMA } & \multicolumn{3}{|c|}{ Long-code CDMA } & \multicolumn{3}{|c|}{ Short-code CDMA } \\
\hline & $200 \mathrm{~m}$ & $500 \mathrm{~m}$ & $1000 \mathrm{~m}$ & $200 \mathrm{~m}$ & $500 \mathrm{~m}$ & $1000 \mathrm{~m}$ & $200 \mathrm{~m}$ & $500 \mathrm{~m}$ & $1000 \mathrm{~m}$ \\
\hline & & & & . & $K=2$ & & - & & \\
\hline Average BER & 0 & 0 & 0 & $19 / 30690$ & $4 / 30690$ & 0 & $36 / 30690$ & 0 & 0 \\
\hline Average SNIR (dB) & 7.2 & 11.5 & 13.3 & 7.1 & 11.3 & 13.6 & 6.9 & 11.2 & 13.1 \\
\hline Average BER & $1174 / 61440$ & $980 / 61440$ & $46 / 61440$ & $3124 / 61440$ & $2066 / 61440$ & $1992 / 61440$ & $1954 / 61440$ & $1886 / 61440$ & $1794 / 61440$ \\
\hline Average SINR (dB) & 4.9 & 6.8 & 7.9 & 4.7 & 6.7 & 7.6 & 4.7 & 6.7 & 7.6 \\
\hline
\end{tabular}

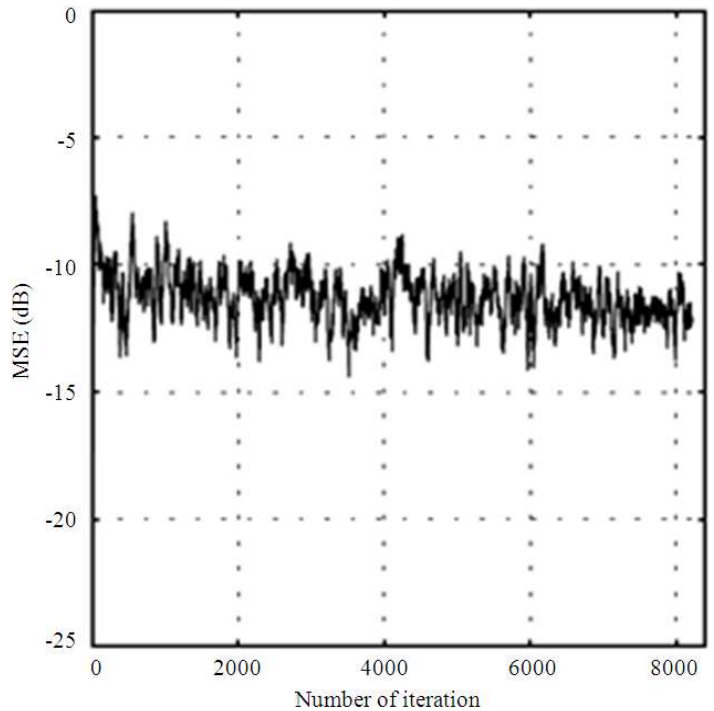

(a)

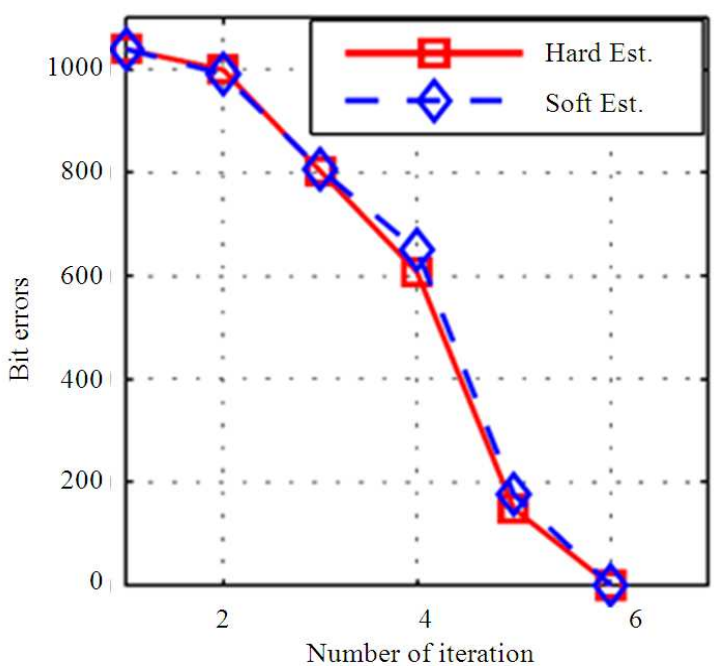

(b)

Fig. 7: Short-code CDMA results, Bit errors $=0$, SINR $=7.1(\mathrm{~dB}),(\mathrm{K}, \mathrm{M}, \mu, \alpha)=(2,16,0.15,0.0001)$ (a) Mean-squared error: (b) Bit errors versus iteration

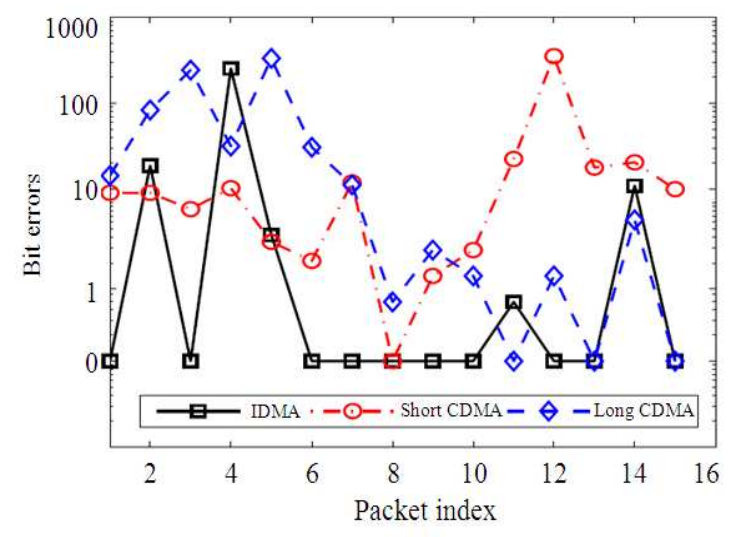

Fig. 8: Bit errors of IDMA, short CDMA and long CDMA averaged over each packet with $\mathrm{K}=4$ over $200 \mathrm{~m}$ channel range

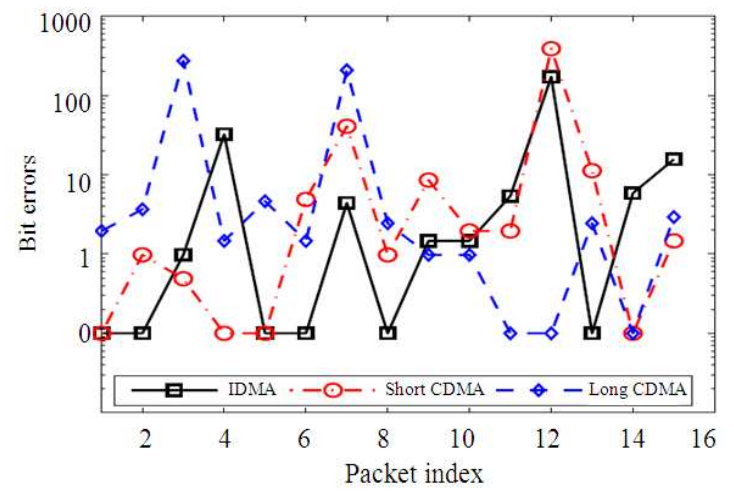

Fig. 9: Bit errors of IDMA, short CDMA and long CDMA averaged over each packet with $K=4$ over $500 \mathrm{~m}$ channel range

The bit error versus iteration number of soft and hard channel estimation are depicted in Fig. 5b-7b. The BERs averaged over each packet are plotted in Fig. 8-10 over the channel ranges 200, 500 and $1000 \mathrm{~m}$, respectively. 
Am. J. Engg. \& Applied Sci., 4 (4): 556-565, 2011

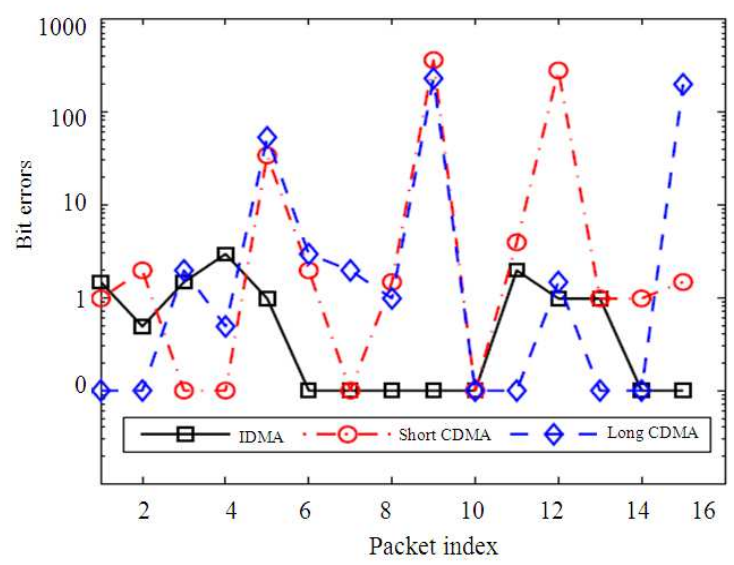

Fig. 10: Bit errors of IDMA, short CDMA and long CDMA averaged over each packet with $K=4$ over $1000 \mathrm{~m}$ channel range

\section{DISCUSSION}

In most packets, the soft channel estimation has less decision feedback errors and rapid convergence speed compared with hard decision channel estimation. These figures also illustrate the number of errors with each pass, where the bit errors reduce dramatically. The receivers have a rapid convergence speed and most of the iterative gains are obtained in the first six iterations. The reason underlying this improvement is due to the redundancy introduced by the spreading and channel coding. The reliable chip decisions help to improve weak chip decisions iteratively until the feedback eventually consists almost entirely of correct chips.

For the $\mathrm{K}=4$ active users scenario, the achieved output SINRs differs significantly. As the number of active user's increases, there are more incorrectly decided symbols and the accuracy of channel estimation will be negatively affected by error propagation, where an error in one decision might lead to errors in subsequent decisions.

The penalty in performance with the $200 \mathrm{~m}$ channel range in almost all the combinations is significant when comparing with the 500 and $1000 \mathrm{~m}$ ranges. The underlying reason for this performance deterioration is due to the channel coefficients to be estimated increasing and the correlation among channel coefficients decreasing due to fading rate. This causes noise in the channel estimate and inaccurate decision feedback symbols which has a higher occurrence at low SINR. Therefore, the channel estimates degrade and the receiver will be not able to reconstruct the MAI and ISI properly. However, a reasonable performance in IDMA over $1000 \mathrm{~m}$ is still achieved, which speaks for the robustness of the iterative receivers. Additionally, the results of the BER and SINR reveal that the IDMA performance is as good as, or slightly outperforms, the long code CDMA and short code CDMA in most configurations. This is due to the fact that in IDMA based systems, the chip level interleaves are more effective in dispersing the bits throughout the packet than their bit level equivalents in CDMA.

\section{CONCLUSION}

IDMA and CDMA turbo multiuser receivers, both employing chip detectors and Rake reception, have been integrated with developed channel estimation and phase tracking and examined for reliable multi-user communication in UACs. The channel estimation was significantly refined by multiplexing the training symbols and multiuser signal. The receivers have been successfully tested and applied to experimental data transmitted over different ranges using QPSK. Extensive results at each scenario were compared and the adaptive IDMA demonstrated slightly improved performances compared to CDMA-based systems. Minor improvements are obtained using soft feedback decisions instead of hard feedback decisions. Additionally, the iterative channel estimation is shown to be robust against severe channel fluctuation and it is limited by MAI especially with small processing gain. Therefore, the feasibility of using iterative receivers is worthy of further research as a strong candidate for UACs.

\section{REFERENCES}

Aliesawi, S., C.C. Tsimenidis, B.S. Sharif and M. Johnston, 2010a. Efficient channel estimation for chip multiuser detection on underwater acoustic channels. Proceedings of the 7th IEEE International Symposium on Communication Systems Networks and Digital Signal Processing (CSNDSP), Jul. 21-23, IEEE Xplore Press, Newcastle Upon Tyne, pp: 173-177.

Aliesawi, S., C.C. Tsimenidis, B.S. Sharif and M. Johnston, 2010b. Performance comparison of IDMA receivers for underwater acoustic channels. Proceedings of the 7th International Symposium on Wireless Communication System (ISWCS), Sept. 19-22, IEEE Xplore Press, York, pp: 596-600. DOI: 10.1109/ISWCS.2010.5624402

Choi, J., 1999. Multipath CDMA channel estimation by jointly utilising pilot and traffic channels. Proc. IEE Commun., 146: 312-318. DOI: 10.1049/ipcom: 19990622

Eggen, T.H., A.B. Baggeroer and J.C. Preisig, 2000. Communication over Doppler spread channels. Part I: Channel and receiver presentation. IEEE J. Ocean. Eng., 25: 62-71. DOI: 10.1109/48.820737 
Eggen, T.H., J.C. Preisig and A.B. Baggeroer, 2001. Communication over Doppler spread channels. II. Receiver characterization and practical results. IEEE J. Ocean. Eng., 26: 612-620. DOI: 10.1109/48.972101

Flanagan, M.F. and A.D. Fagan, 2007. Iterative channel estimation, equalization and decoding for pilotsymbol assisted modulation over frequency selective fast fading channels. IEEE Trans. Vehicular Technol., 56: 1661-1670. DOI: 10.1109/TVT.2007.897215

Haykin, S.S., 2002. Adaptive Filter Theory. 4th Edn., Pearson Education India, ISBN-10: 9788131708699, pp: 936.

Jianguo, H., H. Jing and S. Wei, 2007. A multiuser chip-rate equalization algorithm for CDMA underwater communication systems. Proceedings of the IET Conference on Wireless, Mobile and Sensor Networks, Dec. 12-14, IEEE Xplore Press, Shanghai, China, pp: 1009-1012.

Krzymien, L., D. Truhachev, C. Schlegel and M. Burnashev, 2008. Two-stage detection of partitioned random CDMA. Eur. Trans. Telecommun., 19: 499-509. DOI: 10.1002/ett.1269

Li, H., S.M. Betz and H.V. Poor, 2007. Performance Analysis of iterative channel estimation and multiuser detection in multipath DS-CDMA channels. IEEE Trans. Signal Proc., 55: 1981-1993. DOI: 10.1109/TSP.2007.893229

Li, W. and J.C. Preisig, 2007. Estimation of rapidly time-varying sparse channels. IEEE J. Ocean. Eng., 32: 927-939. DOI: 10.1109/JOE.2007.906409

Milewski, A., 1983. Periodic sequences with optimal properties for channel estimation and fast start-up equalization. IBM J. Res. Dev., 27: 426-431. DOI: 10.1147/rd.275.0426

Moher, M., 1998. An iterative multiuser decoder for near-capacity communications. IEEE Trans. Commun., 46: 870- 880. DOI: 10.1109/26.701309

Nagy, O., M.C. Reed and Z. Shi, 2008. Performance analysis of a generic system model for uncoded IDMA using serial and parallel interference cancellation. Eur. Trans. Telecommun., 19: 511522. DOI: $10.1002 /$ ett. 1268

Nowsheen, N., C. Benson and M. Frater, 2010. A high data-rate, software-defined underwater acoustic modem. Proceedings of the OCEANS, Sept. 20-23, IEEE Xplore Press, Seattle, WA., pp: 1-5. DOI: 10.1109/OCEANS.2010.5664474

Otnes, R. and M. Tuchler, 2004. Iterative channel estimation for turbo equalization of time-varying frequency-selective channels. IEEE Trans. Wireless Commun., 3: 1918-1923. DOI: 10.1109/TWC.2004.837421
Otnes, R. and T.H. Eggen, 2008. Underwater acoustic communications: Long-term test of turbo equalization in shallow water. IEEE J. Ocean. Eng., 33: 321-334. DOI: 10.1109/JOE.2008.925893

Ping, L., 2005. Interleave-division multiple access and chip-by-chip iterative multi-user detection. IEEE Commun. Mag., 43: S19-S23. DOI: 10.1109/MCOM.2005.1452830

Ping, L., L. Liu, K.Y. Wu and W.K. Leung, 2006. Interleave division multiple-access. IEEE Trans. Wireless Commun., 5: 938-947. DOI: 10.1109/TWC.2006.1618943

Proakis, J.G. and M. Salehi, 2008. Digital Communications. 5th Edn., McGraw Hill, Boston, ISBN-10: 0071263780, pp: 1150.

Schoeneich, H. and P.A. Hoeher, 2006. Iterative pilotlayer aided channel estimation with emphasis on interleave-division multiple access systems. EURASIP J. Applied Signal Proc. DOI: 10.1155/ASP/2006/81729

Song, S., A.C. Singer and K.M. Sung, 2004. Soft input channel estimation for turbo equalization. IEEE Trans. Signal Proc., 52: 2885-2894. DOI: 10.1109/TSP.2004.834270

Stojanovic, M. and L. Freitag, 2006. Multichannel detection for wideband underwater acoustic CDMA communications. IEEE J. Ocean Eng., 31: 685-695. DOI: $10.1109 /$ JOE.2006.880389

Stojanovic, M. and Z. Zvonar, 1996. Multichannel processing of broad-band multiuser communication signals in shallow water acoustic channels. Trans. Oceanic Eng., 21: 156-166. DOI: 10.1109/48.486791

Strom, E.G. and S.L. Miller, 2002. Iterative demodulation and channel estimation of orthogonal signaling formats in asynchronous DS-CDMA systems. IEICE Trans. Elect., E85-C: 442-451.

Tchamkerten, A. and I.E. Telatar, 2006. On the use of training sequences for channel estimation. Proceedings of the International Symposium on Information Theory, Sept. 4-9, IEEE Xplore Press, Adelaide, SA., pp: 1391-1395. DOI: 10.1109/ISIT.2005.1523571

Wang, X. and H.V. Poor, 1999. Iterative (turbo) soft interference cancellation and decoding for coded CDMA. IEEE Trans. Commun., 47: 1046-1061. DOI: $10.1109 / 26.774855$

Widrow, B., J. McCool and M. Ball, 1975. The complex LMS algorithm. Proc. IEEE, 63: 719-720. DOI: 10.1109/PROC.1975.9807 
Am. J. Engg. \& Applied Sci., 4 (4): 556-565, 2011

Xiong, X. and J. Hu, 2008. A simplified downlink transmission and receiving scheme for IDMA. Proceedings of the International Conference on Communications, Circuits and Systems, May 2527, IEEE Xplore Press, Fujian, pp: 157-161. DOI: 10.1109/ICCCAS.2008.4657749
Zhou, X., Z. Shi and M.C. Reed, 2007. Iterative channel estimation for IDMA systems in time-varying channels. Proceedings of the IEEE Global Telecommunications Conference, Nov. 26-30, IEEE Xplore Press, Washington DC., pp: 40204024. DOI: 10.1109/GLOCOM. 2007.764 